\title{
Facile Fabrication of Diatomite-Supported ZIF-8 Composite for Solid-Phase Extraction of Benzodiazepines in Urine Samples Prior to High-Performance Liquid Chromatography
}

\author{
Li Du ${ }^{1}$, Shaonan $\mathrm{Xu}{ }^{1}$, Han $\mathrm{Wu}^{1}{ }^{1}$, Tengwen Zhao ${ }^{1}$, Xuesheng Wang ${ }^{1,2, *}$ and Manman Wang ${ }^{1, * \mathbb{D}}$ \\ 1 School of Public Health, North China University of Science and Technology, Tangshan 063210, China; \\ duli27@163.com (L.D.); xvshaonanxsn@163.com (S.X.); wuhan246@126.com (H.W.); \\ ztwen_hbu@163.com (T.Z.) \\ 2 Hebei Province Key Laboratory of Occupational Health and Safety for Coal Industry, School of Public Health, \\ North China University of Science and Technology, Caofeidian, Tangshan 063210, China \\ * Correspondence: xswang64@163.com (X.W.); mmwangheuu@126.com (M.W.); \\ Tel.: +86-031-5880-5576 (X.W. \& M.W.)
}

check for updates

Citation: Du, L.; Xu, S.; Wu, H.; Zhao, T.; Wang, X.; Wang, M. Facile Fabrication of Diatomite-Supported ZIF-8 Composite for Solid-Phase Extraction of Benzodiazepines in Urine Samples Prior to HighPerformance Liquid Chromatography. Molecules 2021, 26, 5209. https:// doi.org/10.3390/molecules26175209

Academic Editor: Hiroyuki Kataoka

Received: 20 July 2021

Accepted: 24 August 2021

Published: 27 August 2021

Publisher's Note: MDPI stays neutral with regard to jurisdictional claims in published maps and institutional affiliations.

Copyright: (c) 2021 by the authors. Licensee MDPI, Basel, Switzerland. This article is an open access article distributed under the terms and conditions of the Creative Commons Attribution (CC BY) license (https:/ / creativecommons.org/licenses/by/ $4.0 /)$.

\begin{abstract}
A novel diatomite-supported zeolitic imidazolate framework-8 sorbent (ZIF-8@Dt-COOH) was in situ fabricated and developed for solid-phase extraction of three benzodiazepines (triazolam, midazolam and diazepam) in urine followed by high-performance liquid chromatography. ZIF$8 @ \mathrm{Dt}-\mathrm{COOH}$ was easily prepared by coating ZIF-8 on the surface of Dt-COOH and characterized by Fourier transform infrared spectra, $X$-ray powder diffractometry and scanning electron microscopy. Compared with bare Dt-COOH, the extraction efficiency of ZIF-8@Dt-COOH for the target was significantly increased from $20.1-39.0 \%$ to $100 \%$. Main extraction parameters, including ionic strength and $\mathrm{pH}$ of solution, loading volume, washing solution, elution solvent and elution volume, were optimized in detail. Under optimum conditions, the developed method gave linearity of three BZDs in $2-500 \mathrm{ng} / \mathrm{mL}(r \geq 0.9995)$. Limits of detection $(S / N=3)$, and limits of quantification $(S / N=10)$ were $0.3-0.4 \mathrm{ng} / \mathrm{mL}$ and $1.0-1.3 \mathrm{ng} / \mathrm{mL}$, respectively. In addition, the average recoveries at three spiked levels $(5,10$ and $20 \mathrm{ng} / \mathrm{mL}$ ) varied from $80.0 \%$ to $98.7 \%$, with the intra-day and inter-day precisions of $1.4-5.2 \%$ and $1.5-8.2 \%$, respectively. The proposed method provided an effective purification performance and gave the enrichment factors of 24.0-29.6. The proposed method was successfully employed for the accurate and sensitive determination of benzodiazepines in urine.
\end{abstract}

Keywords: benzodiazepines; diatomite; zeolitic imidazolate framework-8; solid-phase extraction; urine

\section{Introduction}

Benzodiazepines (BZDs, Figure 1), as a class of psychoactive drugs, are extensively prescribed in the therapy of insomnia, anxiety and convulsive attacks due to their hypnotic, anxiolytic, anticonvulsant and muscle-relaxant properties [1]. However, long-term use of BZDs leads to the risk of dependence, memory loss, fainting and cognitive disturbance [2,3]. Besides therapeutic usage, BZDs with alcohol and other drugs (sedatives, antidepressants and neuroleptics) may result in illicit drug abuse and even drug-facilitated assault cases and robbery [4,5]. Consequently, the accurate and sensitive monitoring of BZDs in pharmaceutical preparations, clinical or criminal examinations is of particular importance.

For biological analysis, sample pretreatment is an important process to isolate desired components and remove matrix interferences from complex matrices [6]. Numerous sample pretreatment techniques have been developed for the determination of BZDs in biofluids, such as liquid-liquid extraction [7], solid-phase microextraction (SPME) [8], magnetic solid-phase extraction (MSPE) [9] and solid-phase extraction (SPE) [10,11]. 


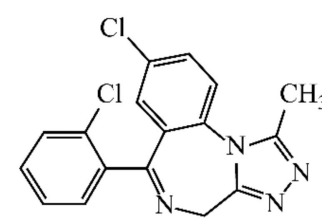

Triazolam

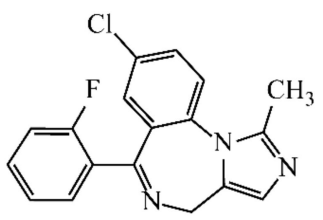

Midazolam

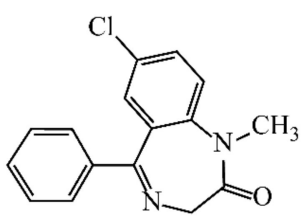

Diazepam

Figure 1. Chemical structures of three BZDs.

SPE is an attractive sample pretreatment methodology owing to the advantages of its high extraction efficiency, low solvent consumption and simplicity in operation [12,13]. Adsorbents in SPE, which play key roles in purification and isolation ability for trace analytes from complex matrices, have attracted increasing interest. Therefore, besides commercial SPE adsorbents $\mathrm{C}_{8}, \mathrm{C}_{18}$, etc., various novel carbon nanotubes, graphene, molecular imprinting polymers and covalent organic frameworks have been explored for sample pretreatment in food, biological, environmental and pharmaceutical analysis [14-17].

Metal-organic frameworks (MOFs) are an attractive coordination polymer constructed with metal ions and organic linkers by coordination bonds [18]. Their extraordinary properties, such as good thermal and mechanical stability, tunable pore topologies and high surface area, make MOFs attractive in the fields of separation, catalysis and sensing [19-21]. Additionally, the utilization of MOFs has attracted intensive attention in sample pretreatment with the mode of SPE, MSPE, dispersive solid-phase extraction (DSPE) and SPME [22-25]. Recently, ZIF-8@cellulose was prepared by simple hydrothermal synthesis and was used as an SPE adsorbent to analyze polycyclic aromatic hydrocarbons in the environment water. The developed method exhibited high sensitivity with a low limit of detection of $0.1-1.0 \mathrm{ng} / \mathrm{L}$ [22]. A novel $\mathrm{Fe}_{3} \mathrm{O}_{4}-\mathrm{NH}_{2} @ \mathrm{MOF}-235$ sorbent was proposed for the extraction of five benzoylurea insecticides from food and environmental samples, including honey, fruit juice and tap water [23]. ZIF-67 was synthesized and employed as a DSPE sorbent for monitoring of buprenorphine in biological fluids coupled with UHPLC-UV [24]. In addition, UiO-67-coated SPME fibers with good thermal stability were applied for the pretreatment of nitrobenzene compounds in environmental water samples before GC-MS [25].

Diatomite (Dt) composed of amorphous siliceous $\left(\mathrm{SiO}_{2} \cdot n \mathrm{H}_{2} \mathrm{O}\right)$ is a kind of natural mineral, and exhibits ordered pore-size distribution and high porosity, as well as exceptional thermal, mechanical and chemical stabilities [26,27]. In addition, the abundance and availability of Dt have attracted particular interest due to its unique properties in the field of catalysis, filtration and adsorption [28-30]. For example, Dt was selected as an environmentally friendly support for the synthesis of $\mathrm{Pd}-\mathrm{M} / \mathrm{Dt}$ to catalyze hydrogenation of long-chain aliphatic esters [28]. A porous Dt ceramic was fabricated and used as an adsorbent for the removal of volatile organic compounds [29]. Additionally, an allophane/Dt nanocomposite with hierarchically porous structure was in situ synthesized for benzene removal [30].

Herein, diatomite-supported ZIF-8 (ZIF-8@Dt-COOH) combining the advantages of both Dt and ZIF was synthesized via simple in situ coating of ZIF-8 on the surface of $\mathrm{Dt}-\mathrm{COOH}$ and applied as an SPE sorbent for isolation of BZDs in urine samples. ZIF-8, as a typical MOF material, is self-assembled from zinc nitrate hexahydrate metal ion and 2-methylimidazole (2-MeIM) ligands with a large surface area, and mechanical and chemical stability [31]. Recently, magnetic ZIF-8 sorbent, carbon and ZIF-8 composite-based membrane/adsorbent have been developed for the pretreatment of tetracycline antibiotics, caffeine and methamphetamine in water, caffeine beverages and urine samples [32-34]. In this work, a novel ZIF-8@Dt-COOH SPE sorbent was prepared and characterized via Fourier transform infrared spectra, $X$-ray powder diffractometry and scanning electron microscopy. The possible mechanism between ZIF-8@Dt-COOH and BZDs was discussed, and potential factors influencing the SPE process were also investigated in detail. The 
developed method of ZIF-8@Dt-COOH-based SPE coupled with HPLC was used for the determination of three BZDs in urine.

\section{Results and Discussion}

\subsection{Synthesis and Characterization of ZIF-8@Dt-COOH}

The ZIF-8 nanocrystals grown on Dt-COOH surface provide the interaction sites, and their density dominates the adsorption efficiency for the target compounds. In order to obtain ZIF-8@Dt-COOH with adequate dense and uniform morphology of ZIF-8, two parameters of $\mathrm{Zn}^{2+}$ concentration $(0.04,0.08$ and $0.12 \mathrm{mmol} / \mathrm{mL})$ and carboxylation cycle of $\mathrm{Dt}(1$ and 2) were optimized. SEM images in Figure 2 show that when the concentration of $\mathrm{Zn}^{2+}$ is fixed at $0.04 \mathrm{mmol} / \mathrm{mL}$, almost no growth of ZIF- 8 crystals is found on the surface of both $\mathrm{Dt}-\mathrm{COOH}(1 \times)$ and $\mathrm{Dt}-\mathrm{COOH}(2 \times)$. As $\mathrm{Zn}^{2+}$ concentration reaches $0.08 \mathrm{mmol} / \mathrm{mL}$, regular dodecahedron ZIF-8 nanocrystals with an average particle diameter of $300-400 \mathrm{~nm}$ are clearly observed on Dt-COOH $(1 \times)$ and Dt-COOH $(2 \times)$. The density of ZIF-8 particles alters with the increase in growth cycles of carboxylation (Figure 2a-f), and more ZIF-8 nanocrystals form on Dt-COOH $(2 \times)$ microspheres than $\mathrm{Dt}-\mathrm{COOH}(1 \times)$, which can be attributed to adequate carboxyl groups providing more affinity sites for the anchoring of $\mathrm{Zn}^{2+}$. Therefore, the concentration of $\mathrm{Zn}^{2+}$ was fixed at $0.08 \mathrm{mmol} / \mathrm{mL}$, and the cycle of carboxylation of Dt was selected to be 2. In this work, the in situ growth of ZIF-8 on $\mathrm{Dt}-\mathrm{COOH}(2 \times)$ was achieved with mechanical stirring in a $70^{\circ} \mathrm{C}$ water bath for $20 \mathrm{~min}$, offering a convenient and fast synthesis.

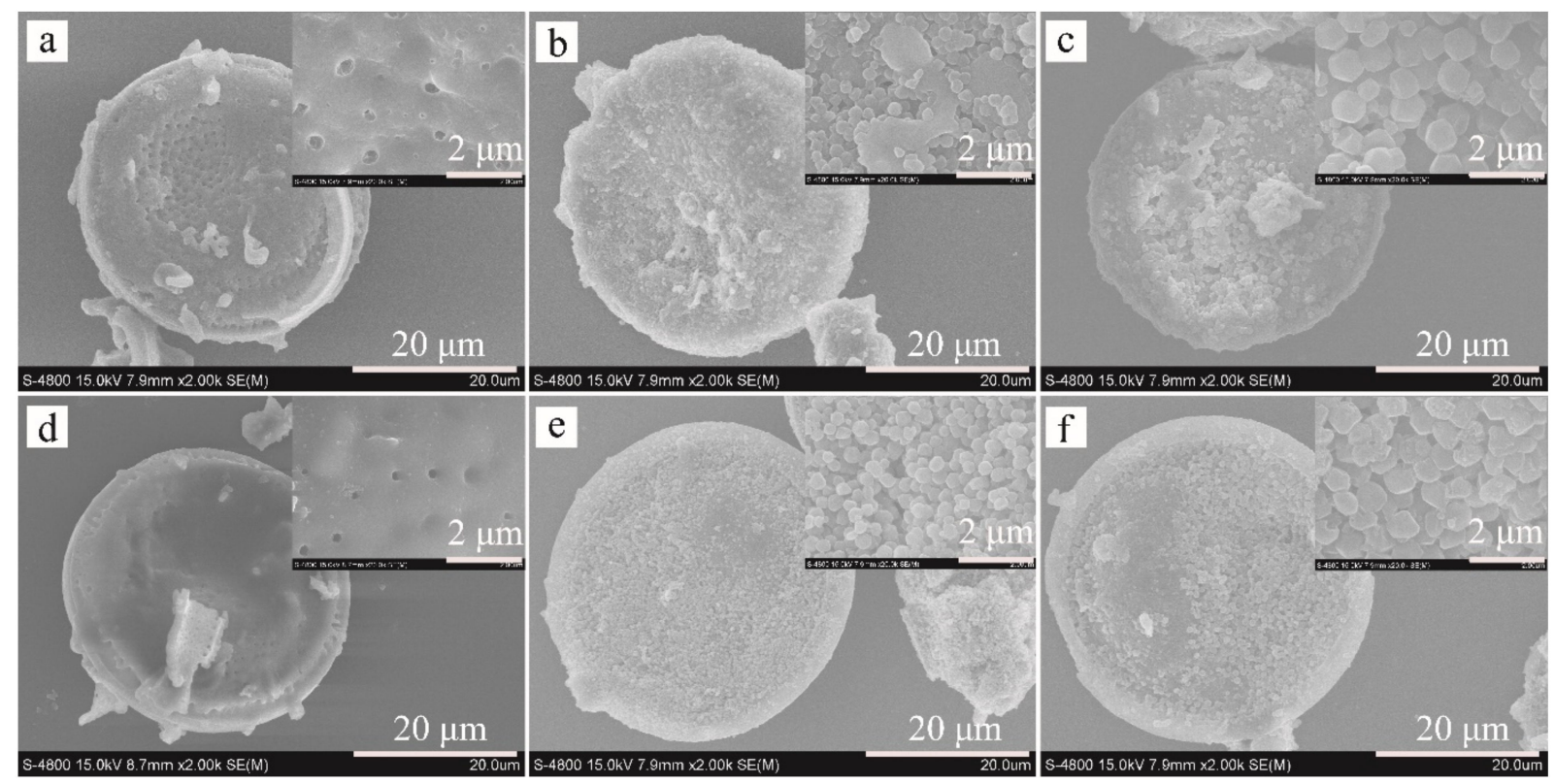

Figure 2. SEM images of $(\mathbf{a}-\mathbf{c}) \mathrm{ZIF}-8 @ \mathrm{Dt}-\mathrm{COOH}(1 \times)$ and $(\mathbf{d}-\mathbf{f}) \mathrm{ZIF}-8 @ \mathrm{Dt}-\mathrm{COOH}(2 \times)$ with $\mathrm{Zn}^{2+}$ concentrations of $0.04,0.08$ and $0.12 \mathrm{mmol} / \mathrm{mL}(2000 \times$ and inlets, $20,000 \times)$.

ZIF-8@Dt-COOH $(2 \times)$ was further characterized by FT-IR and XRD. Figure 3a shows the FT-IR spectra of ZIF-8, Dt-COOH $(2 \times)$ and ZIF-8@Dt-COOH $(2 \times)$. The main peaks appearing at 2926, 1600, 1145 and $995 \mathrm{~cm}^{-1}$ are caused by $\mathrm{C}-\mathrm{H}, \mathrm{C}=\mathrm{N}$ and $\mathrm{C}-\mathrm{N}$ vibration of the imidazole ring in ZIF-8 crystals [35]. The typical peaks of Dt-COOH $(2 \times)$ at 1090, 795 and $616 \mathrm{~cm}^{-1}$ are ascribed to in-plane Si-O-Si vibration, Si-O deformation and Al-O stretching, respectively [36]. Both characteristic signals of ZIF- 8 and Dt- $\mathrm{COOH}(2 \times)$ are observed in the prepared ZIF-8@Dt-COOH $(2 \times)$. In addition, as shown in Figure $3 \mathrm{~b}$, the typical peaks of ZIF-8 at $7.3^{\circ}, 10.3^{\circ}, 12.7^{\circ}, 16.4^{\circ}, 18.0^{\circ}, 24.6^{\circ}$ and $26.7^{\circ}$ and typical peaks of Dt-COOH $(2 \times)$ at $22^{\circ}$ [37] are found in that of ZIF- $@ @ D t-C O O H(2 \times)$. These results demonstrate the successful synthesis of ZIF- 8 on the surface of Dt-COOH $(2 \times)$. 

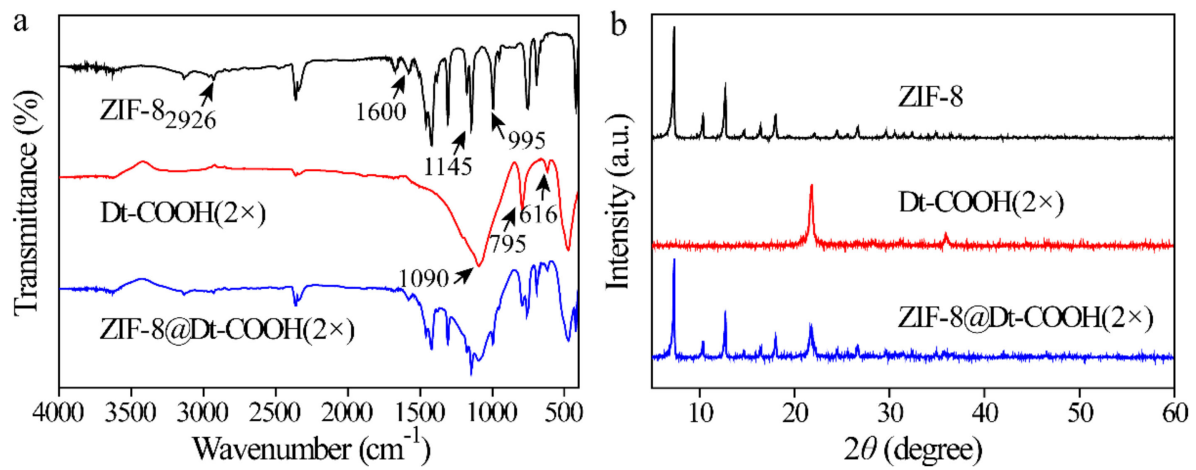

Figure 3. (a,b) FT-IR spectra and XRD patterns of ZIF-8, Dt-COOH $(2 \times)$ and ZIF-8@Dt-COOH( $2 \times)$.

\subsection{Optimization of SPE Conditions}

To achieve high recoveries of BZDs with the prepared ZIF-8@Dt-COOH $(2 \times)$ and enhance the sensitivity of this method, several parameters affecting the SPE performance were investigated, including ionic strength, $\mathrm{pH}$ of solution, loading volume, washing solution, elution solvent and elution volume. Eight milliliters of the spiked aqueous solution (10 ng/mL of each BZD) were loaded onto ZIF-8@Dt-COOH $(2 \times)$ cartridge and all the optimization experiments were repeated three times.

\subsubsection{Ionic Strength and Sample $\mathrm{pH}$}

Ionic strength and $\mathrm{pH}$ of aqueous solution not only affect the molecular states of compounds, but also control the charge species and density of the adsorbent [38]. Consequently, the effect of ionic strength on the recovery was first investigated at an $\mathrm{NaCl}$ concentration of $0-15 \mathrm{mmol} / \mathrm{L}$. Figure $4 \mathrm{a}$ reveals that when the concentration of $\mathrm{NaCl}$ changes from 0 to $15 \mathrm{mmol} / \mathrm{L}$, the recoveries of three BZDs reach $96.6 \pm 4.6 \%-100 \pm 3.8 \%$, revealing that ionic strength has no obvious influence on the adsorption of BZDs. In consideration with the common $\mathrm{pH}$ range of urine samples (4.8-7.4), the $\mathrm{pH}$ of sample solution was also evaluated with $\mathrm{pH}$ 3.8-5.6 (0.1 mmol/L HAc-NaAc) and pH 5.8-7.6 (0.1 mmol/L $\left.\mathrm{Na}_{2} \mathrm{HPO}_{4}-\mathrm{NaH}_{2} \mathrm{PO}_{4}\right)$. The results are shown in Figure $4 \mathrm{~b}$. No significant change in the recoveries of BZDs $(92.5 \pm 5.7 \%-101 \pm 3.2 \%$ ) are observed in the investigated $\mathrm{pH}$ range of 3.8-7.6. In fact, ultrapure water was also explored, and satisfactory extraction was achieved. Thus, ultrapure water was used to dilute the crude urine sample without any adjustment of $\mathrm{pH}$. These results demonstrate that the electrostatic interaction is not the dominant adsorption interaction between ZIF- $8 @ \mathrm{Dt}-\mathrm{COOH}(2 \times)$ and BZDs.
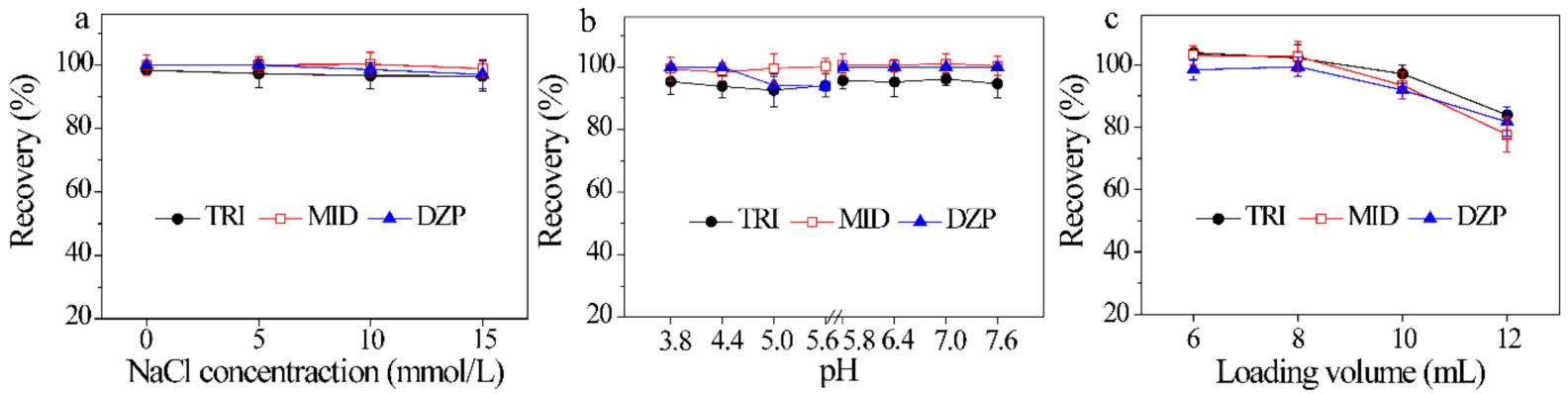

Figure 4. Effects of $(\mathbf{a}-\mathbf{c})$ ionic strength of sample solution, $\mathrm{pH}$ of sample solution and loading volume on the recoveries of three BZDs $(n=3)$.

\subsubsection{Loading Volume}

The loading volume determines the enrichment capacity and sensitivity of the method. The loading volume from $6 \mathrm{~mL}$ to $12 \mathrm{~mL}$ was investigated by spiking the constant amount of BZDs ( $2 \mu \mathrm{g}$ of each analyte) in aqueous solutions. As shown in Figure $4 \mathrm{c}$, the recoveries of BZDs from $98.4 \pm 3.3 \%$ to $104 \pm 2.3 \%$ are obtained with sample volume change from $6 \mathrm{~mL}$ 
to $8 \mathrm{~mL}$. However, further increase in the volume from $10 \mathrm{~mL}$ to $12 \mathrm{~mL}$ leads to a decrease in adsorption efficiencies to $77.6 \pm 4.3 \%$, which are possibly attributed to insufficient contact between BZDs and the adsorbent in large volume. Consequently, the subsequent sample loading volume was carried out with $8 \mathrm{~mL}$.

In addition, the washing process and elution conditions were optimized in detail. The corresponding results and figures are given in the Supporting Information (Figure S1). The optimal conditions were shown as follows: (a) washing, $\mathrm{NaH}_{2} \mathrm{PO}_{4}(25 \mathrm{mmol} / \mathrm{L}, \mathrm{pH}=5)$; (b) elution, $4 \mathrm{~mL}$ of $\mathrm{MeOH}$.

\subsection{Adsorption Performance of ZIF-8@Dt-COOH(2×) for BZDs}

A comparison with the bare Dt-COOH $(2 \times)$ and commercial sorbents (PLS, CX and $\left.\mathrm{C}_{18}\right)$ was performed under the same conditions $(n=3)$ to validate the adsorption performance of ZIF-8@Dt-COOH $(2 \times)$. One hundred fifty milligrams of the adsorbents were packed into the $6 \mathrm{~mL}$ empty syringe individually. Subsequently, the diluted urine sample (urine $\left./ \mathrm{H}_{2} \mathrm{O}, 3 / 1, v / v\right)(8 \mathrm{~mL})$ was loaded onto the cartridges. Figure 5 illustrates that the adsorption efficiency of BZDs on $\mathrm{Dt}-\mathrm{COOH}(2 \times)$ ranges from $20.1 \%$ to $39.0 \%$, while ZIF-8@Dt-COOH $(2 \times)$ provides the significantly enhanced adsorption efficiency of $100 \%$. The results indicate that the presence of ZIF-8 nanoparticles provides the dominant adsorption for the analytes. In addition, ZIF-8@Dt-COOH $(2 \times)$ has superior or comparable adsorption efficiency with the commercial sorbents PLS (100.0\%), CX (94.5-100.0\%), and $\mathrm{C}_{18}(97.6-100 \%)$.

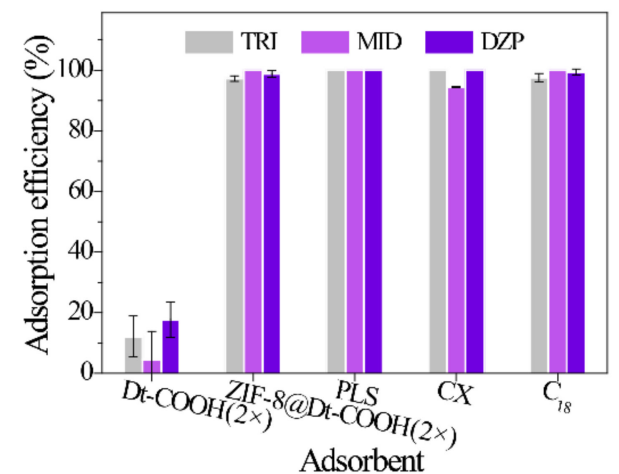

Figure 5. Effect of different SPE sorbents on the adsorption efficiency of three BZDs $(n=3)$.

$\log P$ (octanol-water partition coefficient) refers to the ratio of the solubility of a compound in octanol/water to assess the hydrophobicity of a compound. The higher $\log P$ value, the stronger the hydrophobicity of the compound. The enhancement factor (EF) of all BZDs was calculated by comparing the extracted analytes concentration with that in the original urine sample. As shown in Table 1, DZP with the highest $\log P$ value (4.02) offers an EF of 30.0, while TRI with the lowest $\log P$ value (3.67) gives an EF of 27.8, indicating that the hydrophobic interaction between ZIF-8@Dt-COOH $(2 \times)$ and BZDs play the key role during the adsorption process given by the hydrophobic characteristics of ZIF-8 [39]. Additionally, the $\pi-\pi$ interaction between the aromatic rings of the BZDs and the ZIF- 8 framework is another probable reason for the adsorption.

\subsection{Method Evaluation}

Under the optimal conditions, the developed method was investigated in terms of linearity, correlation coefficients $(r)$, limit of detection (LOD), limit of quantification (LOQ), accuracy and precision. The standard calibration curves were constructed with standard solutions at $2,10,25,50,100,250$ and $500 \mathrm{ng} / \mathrm{mL}(n=3)$. Table 2 shows that the method exhibits linearity ranging from $2-500 \mathrm{ng} / \mathrm{mL}$ with correlation coefficients $(r \geq 0.9995)$ for three BZDs. LODs and LOQs of all analytes in spiked urine samples vary in the range of $0.3-0.4 \mathrm{ng} / \mathrm{mL}$ and $1.0-1.3 \mathrm{ng} / \mathrm{mL}$, respectively. Considering that the excretion concentration of BZDs in urine after clinical administration is approximately $10 \mathrm{ng} / \mathrm{mL}$, 
the recoveries of BZDs at 5, 10 and $20 \mathrm{ng} / \mathrm{mL}$ concentrations in blank urine samples were used to assess the accuracy of this method and the average recoveries of three BZDs varied from $80.0 \%$ to $98.7 \%$. The intra-day and inter-day precisions (relative standard deviations, RSDs) for three replicate extractions of BZDs were $1.4-5.2 \%$ and $1.5-8.2 \%$, respectively. The EFs of all BZDs ranged from 24.0 to 29.6 when the loading volume of the diluted urine sample (urine $/ \mathrm{H}_{2} \mathrm{O}, 3 / 1, v / v$ ) was fixed at $8 \mathrm{~mL}$.

Table 1. $\log P$ and $\mathrm{pKa}$ of the analytes and EF, adsorption efficiency of the proposed materials for BZDs.

\begin{tabular}{|c|c|c|c|c|c|}
\hline \multirow{2}{*}{ Analyte } & \multirow{2}{*}{$\log P^{a}$} & \multirow{2}{*}{$\mathrm{p} K a^{\mathrm{b}}$} & \multirow{2}{*}{ EF } & \multicolumn{2}{|c|}{ Adsorption Efficiency (\%) } \\
\hline & & & & Dt-COOH $(2 \times)$ & ZIF-8@Dt-COOH(2×) \\
\hline TRI & 3.67 & 1.5 & 27.8 & 20.1 & 100 \\
\hline MID & 3.76 & 1.7 & 30.0 & 39.0 & 100 \\
\hline $\mathrm{DZP}$ & 4.02 & 3.3 & 30.0 & 36.1 & 100 \\
\hline
\end{tabular}

${ }^{\mathrm{a}} \log P$ was defined as the ratio of octanol/water partition; ${ }^{\mathrm{b}} \mathrm{pKa}$ was defined as the dissociation constant.

Table 2. Linear range, regression equation $(r)$, LOD, LOQ, recovery, precisions and reproducibility of the proposed method.

\begin{tabular}{|c|c|c|c|c|c|c|c|c|c|c|}
\hline \multirow{2}{*}{ Analyte } & \multirow{2}{*}{$\begin{array}{c}\text { Linear Range } \\
(\mathrm{ng} / \mathrm{mL})\end{array}$} & \multirow{2}{*}{$\underset{(r)}{\text { Regression Equation }^{\mathrm{a}}}$} & \multirow{2}{*}{$\begin{array}{l}\text { LOD } \\
\text { (ng/mL) }\end{array}$} & \multirow{2}{*}{$\underset{\text { (ng/mL) }}{\text { LOQ }}$} & \multirow{2}{*}{$\begin{array}{l}\text { Spiked } \\
\text { (ng/mL) }\end{array}$} & \multirow{2}{*}{$\begin{array}{c}\text { Recovery } \\
(\%)\end{array}$} & \multicolumn{2}{|c|}{$\begin{array}{c}\text { Precisions } \\
(\text { RSD, } \%, n=3)\end{array}$} & \multicolumn{2}{|c|}{ Reproducibility $^{b}$} \\
\hline & & & & & & & Intra-Day & Inter-Day & $\begin{array}{c}\text { Column- } \\
\text { to-Column }\end{array}$ & $\begin{array}{c}\text { Batch-to- } \\
\text { Batch }\end{array}$ \\
\hline TRI & $2-500$ & $\begin{array}{c}y=1.1317-5.615 \\
(0.9995)\end{array}$ & 0.3 & 1.0 & $\begin{array}{c}5 \\
10 \\
20\end{array}$ & $\begin{array}{l}92.9 \\
86.8 \\
96.7\end{array}$ & $\begin{array}{l}5.2 \\
1.4 \\
1.5\end{array}$ & $\begin{array}{l}5.7 \\
2.5 \\
1.5\end{array}$ & 3.3 & 6.9 \\
\hline MID & $2-500$ & $\begin{array}{c}y=0.8581 x-3.349 \\
(0.9996)\end{array}$ & 0.4 & 1.3 & $\begin{array}{c}5 \\
10 \\
20\end{array}$ & $\begin{array}{l}98.7 \\
83.9 \\
81.3\end{array}$ & $\begin{array}{l}4.1 \\
3.7 \\
1.9\end{array}$ & $\begin{array}{l}7.3 \\
3.1 \\
4.3\end{array}$ & 6.4 & 8.0 \\
\hline DZP & $2-500$ & $\begin{array}{c}y=1.3859 x-6.372 \\
(0.9996)\end{array}$ & 0.3 & 1.0 & $\begin{array}{c}5 \\
10 \\
20\end{array}$ & $\begin{array}{l}98.0 \\
80.0 \\
86.5\end{array}$ & $\begin{array}{l}2.9 \\
2.6 \\
1.9\end{array}$ & $\begin{array}{l}7.5 \\
2.9 \\
8.2\end{array}$ & 3.8 & 9.4 \\
\hline
\end{tabular}

${ }^{\mathrm{a}} y$, peak area; $x$, mass concentration, $\mathrm{ng} / \mathrm{mL}^{\mathrm{b}}$ the reproducibility was assessed by calculating the column-to-column and batch-to-batch RSDs $(n=3)$ of BZDs.

The column-to-column and batch-to-batch precisions were used to evaluate the reproducibility of ZIF-8@Dt-COOH $(2 \times)$ cartridges (Table 2 ). The diluted urine sample (urine $\left./ \mathrm{H}_{2} \mathrm{O}, 3 / 1, v / v\right)(8 \mathrm{~mL})$ spiked with $20 \mathrm{ng} / \mathrm{mL}$ of each analyte was loaded onto ZIF-8@Dt-COOH $(2 \times)$ cartridges and the RSDs of the recoveries of BZDs were calculated. The column-to-column RSDs of the three ZIF-8@Dt-COOH $(2 \times)$ cartridges from one batch were $3.3-6.4 \%$, and the batch-to-batch RSDs of three parallel batches ranged from $6.9 \%$ to $9.4 \%$, indicating good reproducibility of ZIF-8@Dt-COOH $(2 \times)$.

\subsection{Application to Urine Samples}

Figure 6 shows the representative HPLC chromatograms of the standard solution (300 ng/mL of each analyte) and urine spiked with $10 \mathrm{ng} / \mathrm{mL}$ of each analyte via direct analysis, extracted by ZIF-8@Dt-COOH $(2 \times)$ and commercial CX adsorbents. Compared with the results of direct analysis (Figure 6b), all BZDs were effectively enriched from complex urine samples by ZIF-8@Dt-COOH $(2 \times)$ with EFs of 24.0-29.6 and the impurity intensities were significantly decreased (Figure 6c). Furthermore, the recovery (\%) was evaluated by the following equation:

$$
\text { Recovery }(\%)=\frac{\mathrm{C}_{\text {found }}-\mathrm{C}_{\text {real }}}{\mathrm{C}_{\text {added }}} \times 100
$$

where $\mathrm{C}_{\text {added }}$ is the concentration of the spiked amount of standard. $\mathrm{C}_{\text {real }}$ and $\mathrm{C}_{\text {found }}$ are the detected concentrations of the analyte before and after the addition of standard in the urine samples, respectively. The recoveries of BZDs obtained by ZIF-8@Dt-COOH $(2 \times)$ cartridges and commercial CX adsorbents were all higher than $80.0 \%$ (Figure $6 c, d$ ). These results 
reveal that the developed technique is effective for the purification and preconcentration of BZDs in urine.

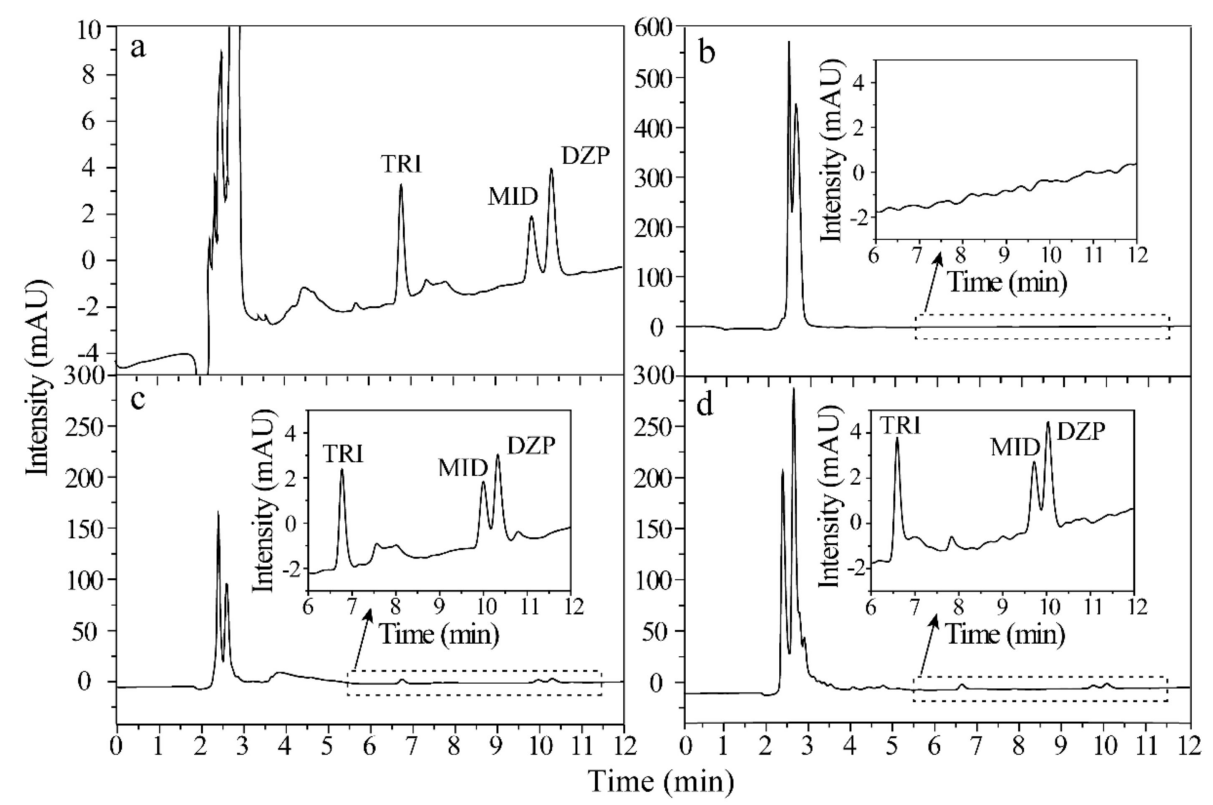

Figure 6. Chromatograms of (a-d) BZDs in the standard solution (300 ng/mL of each analyte), spiked urine sample (10 $\mathrm{ng} / \mathrm{mL}$ of each analyte) with direct injection, pretreated by ZIF-8@Dt-COOH $(2 \times)$ SPE cartridge and pretreated by commercial CX SPE adsorbents.

Table 3 shows a comparison of the proposed method with other techniques for the determination of BZDs in biological fluids. The sensitivity of the present method $(0.3-0.4 \mathrm{ng} / \mathrm{mL})$ is higher than that using an analogous detector, even a mass spectrometry detector (MS) [10,40-45]. Likewise, it also gave lower LODs and comparable recoveries for BZDs than the commercial SPE sorbents of HLB and SLW.

Table 3. Comparison of the proposed method with other methodologies for the determination of BZDs in biological fluids.

\begin{tabular}{|c|c|c|c|c|c|}
\hline Pretreatment Method & Instrument & $\mathrm{EF}^{\mathrm{a}}$ & LOD (ng/mL) & Recovery (\%) & References \\
\hline LLE & LC-MS/MS & $\sim 10$ & $0.01-0.5$ & $81-95$ & [40] \\
\hline SPDE (HLB) ${ }^{b}$ & LC-TOF-MS & $\sim 10$ & 10 & $89.6-105.0$ & [41] \\
\hline SPE (SLW) & UPLC-MS/MS ${ }^{\mathrm{c}}$ & & 0.3 & $65.3-114.3$ & [10] \\
\hline MAE $^{d}$ & HPLC & 5 & $6.2-12.6$ & $89.8-102.1$ & [42] \\
\hline SPE (HLB) & HPLC & 5 & 3 & $68.5-97.6$ & [43] \\
\hline DNUM $^{\mathrm{e}}$ & HPLC & $23.1-24.0$ & $1.2-1.5$ & $92.2-96.0$ & [44] \\
\hline$D-\mu-S P E$ & HPLC & $27.7-32.8$ & $0.2-2.0$ & $84.0-99.0$ & [45] \\
\hline SPE (ZIF-8@Dt-COOH) & HPLC & $24.0-29.6$ & $0.3-0.4$ & $80.0-98.7$ & This work \\
\hline
\end{tabular}

${ }^{\mathrm{a}}$ Enhancement factor, ${ }^{\mathrm{b}}$ hydrophilic-lipophilic balanced copolymer, ${ }^{\mathrm{c}}$ ultra-performance liquid chromatography-tandem mass spectrometry,

$\mathrm{d}$ microwave-assisted extraction, ${ }^{\mathrm{e}}$ dispersive nanomaterial ultrasound-assisted microextraction.

The feasibility of the proposed method was further demonstrated by the analysis of urine samples obtained from the patients taking MID. As listed in Table 4, the concentrations of MID detected in all urine samples are between $10.9 \pm 0.7$ and $21.7 \pm 1.8 \mathrm{ng} / \mathrm{mL}$. The recoveries obtained by spiking $20 \mathrm{ng} / \mathrm{mL}$ MID in urine samples range from $81.1 \%$ to $109 \%$ with RSDs between $3.8 \%$ and $9.9 \%$. These results indicate the availability of the proposed method for the determination of BZDs in urine. 
Table 4. Analytical results of MID in urine samples from the patients administered MID.

\begin{tabular}{cccc}
\hline Sample & Found \pm SD $(\mathbf{n g} / \mathbf{m L})$ & Recovery $^{\mathbf{a}} \mathbf{( \% )}$ & RSD (\%) \\
\hline 1 & $12.4 \pm 1.2$ & 81.1 & 9.9 \\
2 & $10.9 \pm 0.7$ & 94.9 & 6.4 \\
3 & $14.8 \pm 1.2$ & 91.9 & 8.0 \\
4 & $15.1 \pm 1.3$ & 101 & 8.3 \\
5 & $21.4 \pm 0.7$ & 109 & 3.8 \\
6 & $21.7 \pm 1.8$ & 100 & 7.0 \\
\hline
\end{tabular}

a The spiked level was $20 \mathrm{ng} / \mathrm{mL}$ of MID $(n=3)$.

\section{Materials and Methods}

\subsection{Chemicals and Standards}

All reagents used were of analytical grade. Diatomite (Dt, 99.95\%, mean particle size $\sim 30.8 \mu \mathrm{m}$ ), 3-aminopropyltriethoxysilane (APTES), 2-methylimidazole (2-MeIM), zinc nitrate hexahydrate $\mathrm{Zn}\left(\mathrm{NO}_{3}\right)_{2} \cdot 6 \mathrm{H}_{2} \mathrm{O}$ and glutaric anhydride were provided by Aladdin (Shanghai, China). Absolute ethanol was from Tianjin Huihang Chemical Technology Co., Ltd. (Tianjin, China). N,N'-dimethylformamide (DMF), acetone and ammonium acetate $\left(\mathrm{NH}_{4} \mathrm{OAc}\right)$ were acquired from Tianjin Damao Chemical Reagent Factory (Tianjin, China). Acetic acid (HAc), sodium acetate ( $\mathrm{NaAc}$ ), disodium hydrogen phosphate $\left(\mathrm{Na}_{2} \mathrm{HPO}_{4} \cdot 12 \mathrm{H}_{2} \mathrm{O}\right)$, sodium dihydrogen phosphate $\left(\mathrm{NaH}_{2} \mathrm{PO}_{4} \cdot 2 \mathrm{H}_{2} \mathrm{O}\right)$ and sodium chloride $(\mathrm{NaCl})$ were obtained from Tianjin Zhiyuan Chemical Reagent Co., Ltd. (Tianjin, China). Chromatographic-grade methanol (MeOH) and acetonitrile (ACN) were obtained from Fisher Scientific (Geel, Belgium). Ultrapure water was supplied by Hangzhou Wahaha Group Co., Ltd. (Hangzhou, China). Commercial SPE cartridges, containing hydrophilic-lipophilic balance (PLS), cationic-exchange (CX) and $\mathrm{C}_{18}$ were supplied by Dikma (Shanghai, China). The empty polyethylene SPE cartridges (6 mL) were provided by Shanghai Baitaiqi Trading Co., Ltd. (Shanghai, China).

Three benzodiazepine standards containing triazolam (TRI), midazolam (MID) and diazepam (DZP) were from Cerilliant Corp. (Austin, TX, USA). Each stock solution $(1.0 \mathrm{mg} / \mathrm{mL})$ was separately prepared in $\mathrm{MeOH}$. Working solutions were obtained by freshly diluting the corresponding stock solutions with $\mathrm{MeOH}$.

\subsection{Instrumentation}

An Agilent 1260 HPLC-DAD (Agilent Technologies, CA, USA) with G1311B pump system, G1329A auto-sampler, G1316A temperature control center and G1315D DAD detector were used for chromatographic analysis. Scanning electron microscope (SEM) micrographs of the prepared materials were provided by a Hitachi S- 4800 field emission scanning electron microscope (Hitachi, Tokyo, Japan). Fourier transform infrared (FTIR) spectra (4000-400 $\mathrm{cm}^{-1}$ ) were achieved on Shimadzu FT-IR-8400S transform infrared spectrometer (Jasco, Kyoto, Japan). X-ray diffraction (XRD) patterns were acquired by a Brucker D8 Venture single crystal X-ray diffractometer (Bruker, Karlsruhe, Germany).

\subsection{Fabrication of ZIF-8@Dt-COOH}

Figure 7 shows the in situ fabrication process of ZIF-8@Dt-COOH. Carboxylateterminated $\mathrm{Dt}$ particles (Dt-COOH) were used as the support material and prepared according to An et al. [46]. Typically, $1.68 \mathrm{~g}$ of glutaric anhydride was dispersed in $120 \mathrm{~mL}$ of DMF containing APTES (3.48 mL), and the solution was stirred for $3 \mathrm{~h}$ in a water bath at $30{ }^{\circ} \mathrm{C}$. Subsequently, the dispersion solution of $\mathrm{Dt}(2.0 \mathrm{~g})$ in the mixture of DMF $(100 \mathrm{~mL})$ and $\mathrm{H}_{2} \mathrm{O}(9 \mathrm{~mL})$ was added to the above solution. The resultant solution was mechanically stirred for another $5 \mathrm{~h}$ at $30^{\circ} \mathrm{C}$. The collected $\mathrm{Dt}-\mathrm{COOH}$ particles were washed with ultrapure water and absolute ethanol consecutively, and then dried in vacuum overnight at $25^{\circ} \mathrm{C}$. The obtained materials were named as $\mathrm{Dt}-\mathrm{COOH}(1 \times)$ and $\mathrm{Dt}-\mathrm{COOH}(2 \times)$, respectively. 


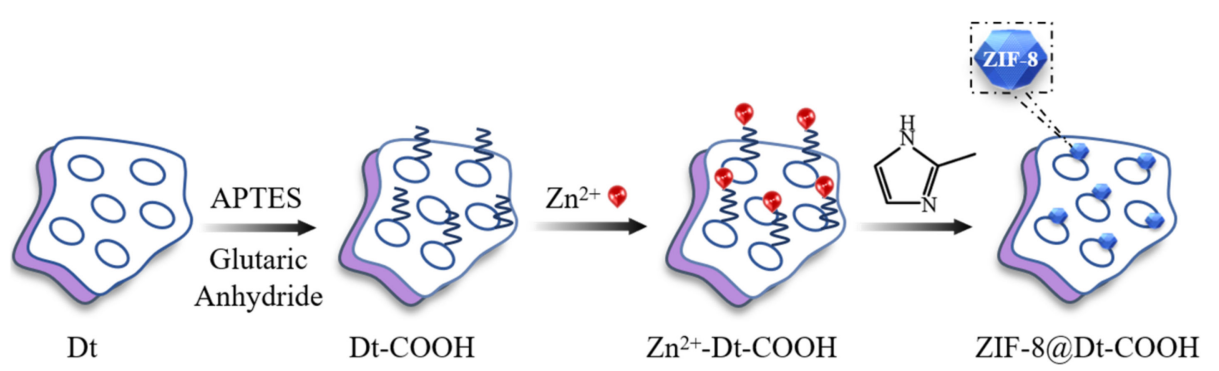

Figure 7. The fabrication flow-chart of ZIF-8@Dt-COOH.

For the growth of ZIF-8 nanocrystals onto Dt-COOH particles, Dt-COOH (1.0 g) was added to $\mathrm{MeOH}(60 \mathrm{~mL})$ solution containing $2.86 \mathrm{~g}$ of $\mathrm{Zn}\left(\mathrm{NO}_{3}\right)_{2} \cdot 6 \mathrm{H}_{2} \mathrm{O}$ and stirred for $5 \mathrm{~min}$ in a $70{ }^{\circ} \mathrm{C}$ water bath. During this process, the affinity of $\mathrm{Zn}^{2+}$ for the carboxyl group results in the adsorption of $\mathrm{Zn}^{2+}$ on the surface of $\mathrm{Dt}-\mathrm{COOH}$ and the obtained $\mathrm{Zn}^{2+} @ \mathrm{Dt}-\mathrm{COOH}$ was used as a precursor for the next reaction. Subsequently, $60 \mathrm{~mL}$ of $\mathrm{MeOH}$ solution containing 7.88 g 2-MeIM was added, and the reaction was maintained for further $15 \mathrm{~min}$ with mechanical stirring at $70{ }^{\circ} \mathrm{C}$. The obtained ZIF-8@Dt-COOH was washed with ultrapure water and absolute ethanol consecutively. Finally, the composite was dried at $60^{\circ} \mathrm{C}$ under vacuum for $3 \mathrm{~h}$. Parameters of $\mathrm{Zn}^{2+}$ concentration $(0.04,0.08$ and $0.12 \mathrm{mmol} / \mathrm{mL}$ ) and carboxylation cycle of Dt (1 and 2) were studied. ZIF-8 nanocrystals were also prepared for comparison.

\subsection{Sample Pretreatment}

This work was approved by the Ethics Committee of North China University of Science and Technology (Tangshan, China). Urine samples were provided by volunteers who were administered MID from North China University of Science and Technology Affiliated Hospital (Tangshan, China). Blank urine samples from healthy volunteers (drugfree) from North China University of Science and Technology were used as controls, and all samples were stored in a refrigerator at $4{ }^{\circ} \mathrm{C}$. The corresponding BZD standards were added to the crude urine fluids to obtain spiked urine samples. Eight milliliters of the diluted urine sample (urine $/ \mathrm{H}_{2} \mathrm{O}, 3 / 1, v / v$ ) was centrifuged at $1500 \mathrm{rpm}$ for $10 \mathrm{~min}$, and the supernatant was selected for analysis.

\subsection{SPE Procedure}

To prepare SPE cartridges, ZIF-8@Dt-COOH $(2 \times)$ adsorbents $(150 \mathrm{mg})$ were packed in the $6 \mathrm{~mL}$ empty syringe and lugged with polypropylene disks to prevent the loss of adsorbent particles. After being preconditioned with $2 \mathrm{~mL}$ of $\mathrm{MeOH}$ and ultrapure water, the cartridge was available for sample pretreatment. The diluted urine sample (urine $/ \mathrm{H}_{2} \mathrm{O}$, $3 / 1, v / v)(8 \mathrm{~mL})$ was loaded onto the cartridge with a flow rate of $0.4 \mathrm{~mL} / \mathrm{min}$. Then, the cartridge was washed with $4 \mathrm{~mL}$ of $\mathrm{NaH}_{2} \mathrm{PO}_{4}$ solution $(25 \mathrm{mmol} / \mathrm{L}, \mathrm{pH}=5)$ and eluted with $4 \mathrm{~mL}$ of $\mathrm{MeOH}$. The collected eluent was evaporated to dryness under $\mathrm{N}_{2}$ gas stream at $35^{\circ} \mathrm{C}$. Finally, the residues were reconstituted with a $0.2 \mathrm{~mL}$ mixture of $\mathrm{MeOH}$ and $\mathrm{H}_{2} \mathrm{O}$ $(65 / 35, v / v)$, and filtered using a $0.22 \mu \mathrm{m}$ filter membrane for HPLC analysis.

Commercial PLS, CX and $\mathrm{C}_{18}$ adsorbents were also employed for the pretreatment of the urine in the optimized conditions for comparison.

\subsection{HPLC Analysis}

HPLC analysis was performed on a $\mathrm{C}_{18}$ column $(250 \mathrm{~mm} \times 4.6 \mathrm{~mm}, 5 \mu \mathrm{m}$, Agilent, CA, USA). The mixture of $\mathrm{MeOH}$ and $\mathrm{H}_{2} \mathrm{O}$ was performed as a mobile phase at a flow rate of $1 \mathrm{~mL} / \mathrm{min}$. The gradient elution procedure was set as $0-9 \mathrm{~min}, 62 \%$ to $70 \% \mathrm{MeOH}$, 9-12 $\mathrm{min}, 70 \% \mathrm{MeOH}$. The detection wavelength was $228 \mathrm{~nm}$, and the injection volume was $20 \mu \mathrm{L}$. 


\section{Conclusions}

In this work, a novel ZIF-8@Dt-COOH composite was synthesized using carboxylateterminated Dt particles as nucleation, $\mathrm{Zn}$ (II) as the central ion and 2-methylimidazole as the ligand by controllable in situ reaction within $20 \mathrm{~min}$. The prepared ZIF-8@Dt-COOH was developed as an SPE adsorbent for isolation of three BZDs in urine, and, compared with the bare Dt-COOH, the extraction efficiency for BZDs was significantly improved from $20.1-39.0 \%$ to $100 \%$. In addition, it exhibited superior extraction performance in comparison with commercial PLS, CX and $C_{18}$ sorbents. The interaction of $\pi-\pi$ and hydrophobicity between BZDs and ZIF-8 are responsible for the high adsorption of BZDs on ZIF-8@Dt$\mathrm{COOH}$ with EFs of 24.0-29.6. These results confirm that the proposed method could be utilized in the preconcentration of BZDs in urine samples.

Supplementary Materials: The following are available online, Figure S1. Effects of $(a-c)$ washing solution, elution solvent and elution volume on the recoveries of three BZDs $(n=3)$.

Author Contributions: Methodology, L.D., S.X., H.W. and T.Z.; Software, L.D., S.X. and H.W.; Validation, L.D., S.X., H.W. and T.Z.; Formal analysis, L.D., S.X., H.W. and T.Z.; Investigation, L.D.; Resources, X.W. and M.W.; Data curation, L.D.; Writing—original draft preparation, L.D.; Writingreview and editing, X.W. and M.W.; Visualization, L.D., S.X., H.W. and T.Z.; Supervision, X.W. and M.W.; Project administration, X.W. and M.W.; Funding acquisition, L.D. and M.W. All authors have read and agreed to the published version of the manuscript.

Funding: The research was financially supported by the National Natural Science Foundation of China (grant number No. 82073608).

Institutional Review Board Statement: The study was conducted according to the guidelines of the Declaration of Helsinki, and approved by Ethics Committee of North China University of Science and Technology $(2021033,2021.02 .27)$.

Informed Consent Statement: Informed consent was obtained from all subjects involved in the study.

Data Availability Statement: The data presented in this study are available on request from the corresponding author.

Conflicts of Interest: The authors declare no conflict of interest.

Sample Availability: Samples of the compounds are not available from the authors.

\section{References}

1. Silva, A.V.D.; Meneghetti, S.M.P.; Meneghetti, M.R. Benzodiazepines: Drugs with chemical skeletons suitable for the preparation of metallacycles with potential pharmacological activity. Molecules 2021, 26, 2796. [CrossRef] [PubMed]

2. Guina, J.; Merrill, B. Benzodiazepines I: Upping the care on downers: The evidence of risks, benefits and alternatives. J. Clin. Med. 2018, 7, 17. [CrossRef] [PubMed]

3. Van Der Sluiszen, N.N.J.J.M.; Vermeeren, A.; Jongen, S.; Vinckenbosch, F.; Ramaekers, J.G. Influence of long-term benzodiazepine use on neurocognitive skills related to driving performance in patient populations: A review. Pharmacopsychiatry 2017, 50, 189-196. [CrossRef] [PubMed]

4. Lader, M. Benzodiazepine harm: How can it be reduced? Br. J. Clin. Pharmacol. 2014, 77, 295-301. [CrossRef]

5. Grigoriadis, S.; Graves, L.; Peer, M.; Mamisashvili, L.; Dennis, C.L.; Vigod, S.N.; Steiner, M.; Brown, C.; Cheung, A.; Dawson, H.; et al. Benzodiazepine use during pregnancy alone or in combination with an antidepressant and congenital malformations: Systematic review and meta-analysis. J. Clin. Psychiatry 2019, 80, 4. [CrossRef] [PubMed]

6. Hashemi, B.; Zohrabi, P.; Shamsipur, M. Recent developments and applications of different sorbents for SPE and SPME from biological samples. Talanta 2018, 187, 337-347. [CrossRef] [PubMed]

7. Furugen, A.; Nishimura, A.; Kobayashi, M.; Umazume, T.; Narumi, K.; Iseki, K. Quantification of eight benzodiazepines in human breastmilk and plasma by liquid-liquid extraction and liquid-chromatography tandem mass spectrometry: Application to evaluation of alprazolam transfer into breastmilk. J. Pharm. Biomed. 2019, 168, 83-93. [CrossRef]

8. Abrão, L.C.D.C.; Figueiredo, E.C. A new restricted access molecularly imprinted fiber for direct solid phase microextraction of benzodiazepines from plasma samples. Analyst 2019, 144, 4320-4330. [CrossRef]

9. Zhang, S.; Yao, W.; Fu, D.; Zhang, C.; Zhao, H. Fabrication of magnetic zinc adeninate metal-organic frameworks for the extraction of benzodiazepines from urine and wastewater. J. Sep. Sci. 2018, 41, 1864-1870. [CrossRef] [PubMed]

10. Zhang, L.; Wu, P.; Jin, Q.; Hu, Z.; Wang, J. Multi-residue analysis of sedative drugs in human plasma by ultra-high performance liquid chromatography tandem mass spectrometry. J. Chromatogr. B 2018, 1072, 305-314. [CrossRef] [PubMed] 
11. Zhao, T.; Du, L.; Zhang, Z.; Li, N.; Wang, M.; Ren, Q. A poly(N,N-dimethylaminoethyl methacrylate-co-ethylene glycol dimethacrylate) monolith for direct solid-phase extraction of benzodiazepines from undiluted human urine. Anal. Methods 2020, 12, 3924-3932. [CrossRef]

12. Andrade-Eiroa, A.; Canle, M.; Leroy-Cancellieri, V.; Cerdà, V. Solid-phase extraction of organic compounds: A critical review. part ii. TRAC-Trends Anal. Chem. 2016, 80, 655-667. [CrossRef]

13. Hansen, F.A.A.; Pedersen-Bjergaard, S. Emerging extraction strategies in analytical chemistry. Anal. Chem. 2019, 92, 2-15. [CrossRef] [PubMed]

14. Nema, T.; Chan, E.C.; Ho, P.C. Applications of monolithic materials for sample preparation. J. Pharm. Biomed. Anal. 2014, 87, 130-141. [CrossRef]

15. Afsharipour, R.; Shabani, A.M.H.; Dadfarnia, S.; Kazemi, E. Selective fluorometric determination of sulfadiazine based on the growth of silver nanoparticles on graphene quantum dots. Microchim. Acta 2020, 187, 54. [CrossRef]

16. Khatibi, S.A.; Hamidi, S.; Siahi-Shadbad, M.R. Current trends in sample preparation by solid-phase extraction techniques for the determination of antibiotic residues in foodstuffs: A review. Crit. Rev. Food Sci. Nutr. 2020, 1-22. [CrossRef]

17. Xin, J.; Wang, X.; Li, N.; Liu, L.; Lian, Y.; Wang, M.; Zhao, R. Recent applications of covalent organic frameworks and their multifunctional composites for food contaminant analysis. Food Chem. 2020, 330, 127255. [CrossRef] [PubMed]

18. Safaei, M.; Foroughi, M.M.; Ebrahimpour, N.; Jahani, S.; Omidi, A.; Khatami, M. A review on metal-organic frameworks: Synthesis and applications. TrAC Trends Anal. Chem. 2019, 118, 401-425. [CrossRef]

19. Li, L.; Chen, Y.; Yang, L.; Wang, Z.; Liu, H. Recent advances in applications of metal-organic frameworks for sample prepa-ration in pharmaceutical analysis. Coordin. Chem. Rev. 2020, 411, 213235. [CrossRef]

20. Rocío-Bautista, P.; Pacheco-Fernández, I.; Pasán, J.; Pino, V. Are metal-organic frameworks able to provide a new generation of solid-phase microextraction coatings?-A review. Anal. Chim. Acta 2016, 939, 26-41. [CrossRef]

21. Wang, Q.; Gu, C.; Fu, Y.; Liu, L.; Xie, Y. Ultrasensitive electrochemical sensor for luteolin based on zirconium metal-organic framework UiO-66/reduced graphene oxide composite modified glass carbon electrode. Molecules 2020, 25, 4557. [CrossRef]

22. Yang, H.; Li, L.; Cao, H.; Zhang, Z.; Zhao, T.; Hao, Y.; Wang, M. Silica supported metal organic framework 808 composites as adsorbent for solid-phase extraction of benzodiazepines in urine sample. Microchem. J. 2020, 157, 105062. [CrossRef]

23. Duo, H.; Lu, X.; Wang, S.; Wang, L.; Guo, Y.; Liang, X. Synthesis of magnetic metal-organic framework composites, $\mathrm{Fe}_{3} \mathrm{O}_{4}-\mathrm{NH}_{2} @ \mathrm{MOF}-235$, for the magnetic solid-phase extraction of benzoylurea insecticides from honey, fruit juice and tap water samples. New J. Chem. 2019, 43, 12563-12569. [CrossRef]

24. Mohammadi, F.; Shabani, A.M.H.; Dadfarnia, S.; Ansari, M.; Asgharinezhad, A.A. Dispersive solid-phase extraction of buprenorphine from biological fluids using metal-organic frameworks and its determination by ultra-performance liquid chromatography. J. Sep. Sci. 2020, 43, 3045-3052. [CrossRef]

25. Manousi, N.; Zachariadis, G.A.; Deliyanni, E.A.; Samanidou, V.F. Applications of metal-organic frameworks in food sample preparation. Molecules 2018, 23, 2896. [CrossRef]

26. Namor, A.F.D.; Elgamouz, A.; Frangie, S.; Martinez, V.; Valiente, L.; Webb, O.A. Turning the volume down on heavy metals using tuned diatomite. A review of diatomite and modified diatomite for the extraction of heavy metals from water. J. Hazard. Mater. 2012, 241-242, 14-31. [CrossRef] [PubMed]

27. He, H.; Luo, Y.; Luo, Z.; Yu, C. Diatomite-based material as an adsorbent or photocatalyst for water treatment. Prog. Chem. 2019, 31, 561-570. [CrossRef]

28. Huang, C.; Zhang, H.; Zhao, Y.; Chen, S.; Liu, Z. Diatomite-supported Pd-M (M = Cu, Co, Ni) bimetal nanocatalysts for selective hydrogenation of long-chain aliphatic esters. J. Colloid Interface Sci. 2012, 386, 60-65. [CrossRef] [PubMed]

29. Wang, S.; Lee, Y.-N.; Nam, H.; Nam, H.; Kim, H.-K. Chemical activation of porous diatomite ceramic filter for the adsorption of TMA, $\mathrm{H}_{2} \mathrm{~S}, \mathrm{CH}_{3} \mathrm{COOH}$ and $\mathrm{NH}_{3}$ : Isotherm and kinetic studies. J. Environ. Chem. Eng. 2019, 7, 103481. [CrossRef]

30. Deng, L.; Du, P.; Yu, W.; Yuan, P.; Annabi-Bergaya, F.; Liu, D.; Zhou, J. Novel hierarchically porous allophane/diatomite nanocomposite for benzene adsorption. Appl. Clay Sci. 2019, 168, 155-163. [CrossRef]

31. Chen, B.; Yang, Z.; Zhu, Y.; Xia, Y. Zeolitic imidazolate framework materials: Recent progress in synthesis and applications. J. Mater. Chem. A 2014, 2, 16811-16831. [CrossRef]

32. Liu, H.; Chen, L.; Ding, J. A core-shell magnetic metal organic framework of type $\mathrm{Fe}_{3} \mathrm{O}_{4} @ Z$ ZIF- 8 for the extraction of tetracycline antibiotics from water samples followed by ultra-HPLC-MS analysis. Microchim. Acta 2017, 184, 4091-4098. [CrossRef]

33. Jafari, Z.; Hadjmohammadi, M.R. In situ growth of zeolitic imidazolate framework-8 on a GO-PVDF membrane as a sorbent for thin-film microextraction of caffeine followed by quantitation through high-performance liquid chromatography. Anal. Methods 2020, 12, 1736-1743. [CrossRef]

34. Taghvimi, A.; Tabrizi, A.B.; Dastmalchi, S.; Javadzadeh, Y. Metal organic framework based carbon porous as an efficient dispersive solid phase extraction adsorbent for analysis of methamphetamine from urine matrix. J. Chromatogr. B 2019, 1109, 149-154. [CrossRef] [PubMed]

35. Venna, S.R.; Jasinski, J.B.; Carreon, M.A. Structural evolution of zeolitic imidazolate framework-8. J. Am. Chem. Soc. 2010, 132, 18030-18033. [CrossRef] [PubMed]

36. Tsai, W.T.; Lai, C.W.; Hsien, K.J. Characterization and adsorption properties of diatomaceous earth modified by hydrofluoric acid etching. J. Colloid Interface Sci. 2006, 297, 749-754. [CrossRef] [PubMed] 
37. Yuan, W.; Yuan, P.; Liu, D.; Deng, L.; Zhou, J.; Yu, W.; Chen, F. A hierarchically porous diatomite/silicalite-1 composite for benzene adsorption/desorption fabricated via a facile premodification in situ synthesis route. Chem. Eng. J. 2016, $294,333-342$. [CrossRef]

38. Rezaei, F.; Yamini, Y.; Moradi, M.; Daraei, B. Supramolecular solvent-based hollow fiber liquid phase microextraction of benzodiazepines. Anal. Chim. Acta 2013, 804, 135-142. [CrossRef] [PubMed]

39. Bayati, B.; Ghorbani, A.; Ghasemzadeh, K.; Iulianelli, A.; Basile, A. Study on the separation of $\mathrm{H}_{2}$ from $\mathrm{CO}_{2}$ using a ZIF-8 membrane by molecular simulation and maxwell-stefan model. Molecules 2019, 24, 4350. [CrossRef]

40. Jang, M.; Chang, H.; Yang, W.; Choi, H.; Kim, E.; Yu, B.-H.; Oh, Y.; Chung, H. Development of an LC-MS/MS method for the simultaneous determination of 25 benzodiazepines and zolpidem in oral fluid and its application to authentic samples from regular drug users. J. Pharm. Biomed. Anal. 2012, 74, 213-222. [CrossRef]

41. Saito, K.; Kikuchi, Y.; Saito, R. Solid-phase dispersive extraction method for analysis of benzodiazepine drugs in serum and urine samples. J. Pharm. Biomed. Anal. 2014, 100, 28-32. [CrossRef] [PubMed]

42. Fernández, P.; Vázquez, C.; Lorenzo, R.A.; Carro, A.M.; Alvarez, I.; Cabarcos, P. Experimental design for optimization of microwave-assisted extraction of benzodiazepines in human plasma. Anal. Bioanal. Chem. 2010, 397, 677-685. [CrossRef] [PubMed]

43. Cabarcos, P.; Tabernero, M.J.; Álvarez, I.; López, P.; Fernández, P.; Bermejo, A.M. Analysis of six benzodiazepines in vitreous humor by high-performance liquid chromatography-photodiode-array detection. J. Anal. Toxicol. 2010, 34, 539-542. [CrossRef] [PubMed]

44. Pebdani, A.A.; Khodadoust, S.; Talebianpoor, M.; Zargar, H.; Zarezade, V. Preconcentration and determination of chlordiazepoxide and diazepam drugs using dispersive nanomaterial-ultrasound assisted microextraction method followed by high performance liquid chromatography. J. Chromatogr. B 2016, 1008, 146-155. [CrossRef] [PubMed]

45. Asgharinezhad, A.A.; Ebrahimzadeh, H.; Mirbabaei, F.; Mollazadeh, N.; Shekari, N. Dispersive micro-solid-phase extraction of benzodiazepines from biological fluids based on polyaniline/magnetic nanoparticles composite. Anal. Chim. Acta 2014, 844, 80-89. [CrossRef]

46. An, Y.; Chen, M.; Xue, Q.; Liu, W. Preparation and self-assembly of carboxylic acid-functionalized silica. J. Colloid Interface Sci. 2007, 311, 507-513. [CrossRef] 